\title{
The Recent Trends in Research on Contact Phenomena in the Railway System
}

\author{
Mitsuru IKEDA \\ Power Supply Technology Division (Formerly, Railway Dynamics Division)
}

\begin{abstract}
Contact phenomena often lie at the heart of solutions to be found for various problems related to the railway system. Clarifying these phenomena is therefore crucial to improve the quality of railway operations. This paper describes research to gather greater insight into an array of contact phenomena such as, rolling contact of wheels and rails, impact motion of vehicles and railway structures, sliding contact between overhead lines and pantographs, etc..
\end{abstract}

Keywords: contact, railway dynamics, dynamics, simulation

\section{Introduction}

Studying dynamic behavior from an engineering angle often raises the problem of how to tackle contact phenomena. This is because contact phenomena are not just complex in themselves, but also stem from multiple factors, which make its particularly difficult to fully understand. In addition, since in many cases contact phenomena have a major impact on the behavior of the entire system being studied, analytical results can vary greatly depending on how contact phenomena are modeled in the analysis. As such, formulation and modeling of contact phenomena require careful thought. These issues all directly apply to the study of railway dynamics.

In ordinary type of railway system, a vehicle moves as its wheels make contact with the rails. The vehicle is also guided laterally thanks to wheel/rail contact. On ballasted track, the weight of a train, several dozen tons per vehicle, is transferred to the roadbed through the crushed stones contacting with each other beneath the rails, thereby efficiently supporting the mass of the train. Furthermore, electrical power is normally supplied to vehicles through sliding contact between the current collector on the vehicle and overhead contact lines. Any problem involving these points of contact can disrupt train operations, which means contact phenomena have long been an important focus for study in the railways. As in the past, efforts continue today to address the many issues related to contact phenomena. This paper describes RTRI's work over recent years to address these matters.

\section{Analysis of rolling contact between wheels and rails}

The Hertzian contact theory has been used to take an engineering approach to the study of contact between two objects, such as between wheels and rails. The theory addresses contact between two objects with curved surfaces, and provides the size and stress distribution of the contact area on the premise that the deformation of the objects in contact occurs within the elastic limits of the objects' materials. In actual rolling contact, slip occurs in part or all of the contact area of the two objects, which in a railway context substantially affects the wheel/rail adhesion char- acteristics [1]. Analyses of this type of contact are often conducted using Kalker's exact rolling contact theory [2]. However, with each wheel of an ordinary vehicle applying a load of around $50 \mathrm{kN}$ on average on the rail, the stress working on the wheel-rail contact surfaces exceeds the elastic limits even at a standstill. In addition, while contact stress demonstrates unsteady behavior, contact analysis taking account of plastic deformation and the unsteady behavior poses many challenges.

Given the above, with the aim of developing a numerical analysis-based method for wheel-rail rolling contact analysis that does not require assumption-based theoretical treatment, RTRI has been involved in a joint study with Professor Okuda of the University of Tokyo to develop a wheel-rail rolling contact simulator [3] capable not just of dealing with material nonlinearity, geometrical nonlinearity and other factors more exactly, but also of performing unsteady, dynamic rolling contact analyses. The simulator, which is based on the large-scale parallel FEM structural analysis software FrontISTR [4], is capable of analyzing dynamic stress distribution on wheel-rail contact surfaces with sufficient resolution by dividing the surfaces into fine mesh. However, the use of fine mesh is accompanied by the need for extremely large-scale computing. Sophisticated use of supercomputers and high-performance computing (HPC) techniques is therefore a must. The simulator program started out with a 1-wheel/1-axle model capable of behaving only as a single wheel on a single rail, moved onto a 2-wheel/2-axle model and is currently at the stage of developing a 4-wheel/2-axle model with which can evaluate the rolling contact of a complete bogie (Fig. 1). With the 2 -wheel/2-axle and earlier models, the movement in the direction of sleepers was constrained. With the 4-wheel/2axle model, this constraint is no longer imposed, making it possible to analyze rolling contact behavior including in curve section (Fig. 1 (c)). As part of efforts to address longterm deterioration of rails caused by wheel-rail rolling contact, much of which occurs in curved sections, a simulator was developed for analyzing cracking and progress of cracking in rails [5]. This analysis is based on quasi-static analysis to impose stress distribution on wheel-rail contact surfaces on crack-progress analysis. As the accuracy of the wheel-rail rolling contact simulator has improved for analysis of the contact stress distribution in curve sections, it will be used to help clarify the mechanisms involved in 

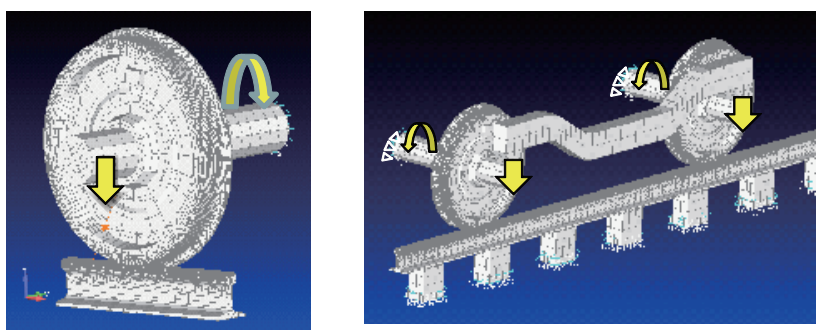

(a) 1-wheel / 1-axle model

(b) 2-wheel / 2-axle model

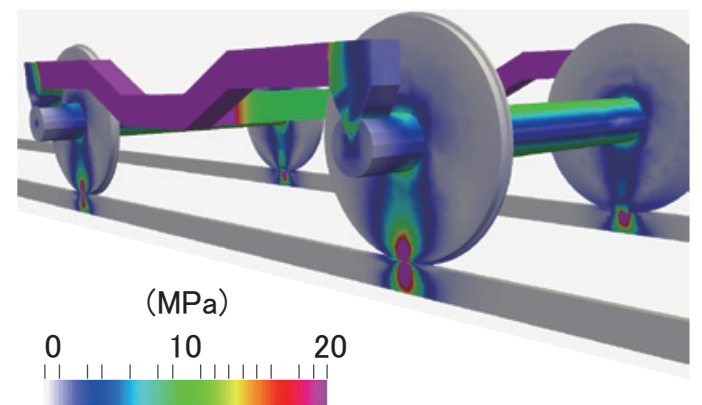

(c) 4-wheel / 2-axle model

(stress analysis when running through a transition curve)

\section{Fig. 1 Wheel-rail rolling contact simulator}

long-term rail deterioration and the causal factors of deterioration.

Rails in open sections may be covered with various kinds of matter that can greatly affect vehicle adhesion if lodged between the wheels and rails, such as fallen leaves. From autumn through early winter, leaves on the track in mountainous sections can cause wheels to slip and slide, forcing train delays or cancellations (Fig. 2). Hence, studies are underway to clarify how fallen leaves reduce adhesion
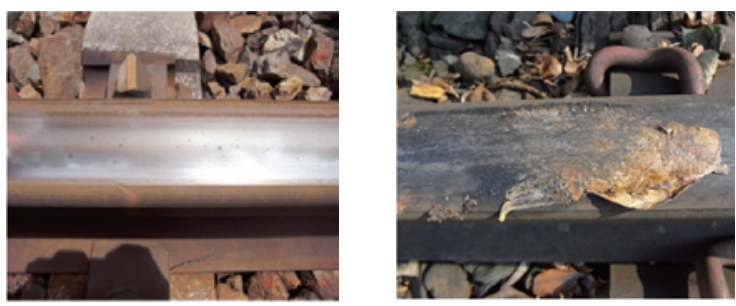

(a) Difference in surface condition of a rail in spring (left) and autumn (right)

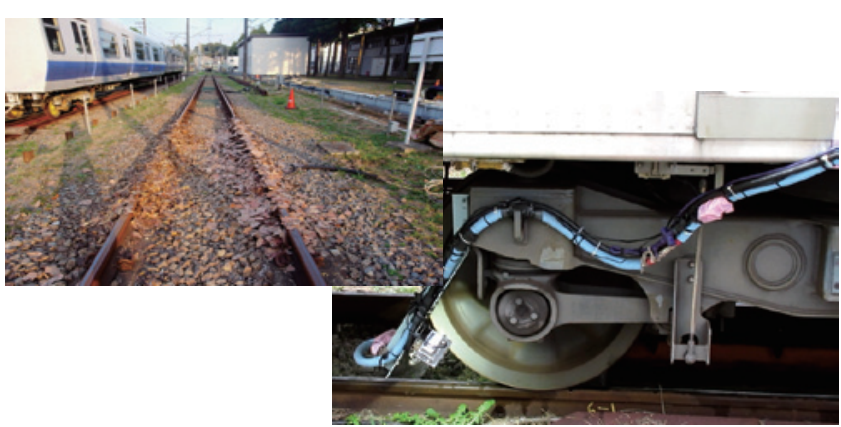

(b) Experiment on RTRI test track to investigate the influence of leaves on adhesion behavior

Fig. 2 Study of the influence of fallen leaves on wheel/ rail adhesion and to develop countermeasures.

Through ongoing field investigations conducted primarily along lines in mountainous areas on the island of Honshu and various kinds of experiments on test tracks and other test apparatus at RTRI, the basic mechanisms leading to loss of adhesion arising from fallen leaves are being clarified. The following is an outline of these mechanisms [6]. As a wheel repeatedly runs over leaves on a rail, leaves are ground to small pieces and adhere to the rail. The tannin contained in this pulp reacts with iron in certain ambient conditions (temperature, humidity) and turns into a compound of iron and tannin and eventually into a black film. In wet and humid conditions, the black film turns into a paste, substantially reducing the adhesion of wheels as they run over it and causing them to slip and slide.

Based on this insight, measures are now being developed to prevent wheel slipping and sliding. In Japan and abroad, some countermeasures against adhesion reduction have already been implemented including high-pressure washing of rails or polishing of rail surfaces. However, the iron and tannin compound at the root of the problem and described earlier is insoluble in water. It would therefore be difficult to remove the compound by just washing. RTRI therefore plans to continue to developing more effective countermeasures.

\section{Contact analysis involving identifying contact pairs}

As discussed earlier in this paper, on ballasted tracks, the load applied by vehicles on the rails is transferred via the crushed stones to the roadbed. Through the load transfer process to the roadbed, the load applied by the vehicle on the rail is dispersed over the roadbed, which means that the ballast is a highly efficient sleeper-supporting structure. However, ballast settles as the crushed stones move and are worn, which requires regular maintenance. RTRI has been advancing study programs to clarify the dynamic behavior of crushed stones in contact with each other in order to develop a new type of ballasted track that requires less maintenance [7, 8]. At the same time, with increasing seismic activity in and around Japan in recent years, it is becoming increasingly important to predict how vehicles behave as they derail because of a massive earthquake. With the above in mind, RTRI has conducted derailment experiments using an actual bogie on a large-scale shaking table [9] as well as numerical simulations of vehicle behavior during derailment $[10,11]$.

An engineering challenge common to these analyses is how efficiently contact pairs, which are important in contact analysis, are identified. When analyzing the dynamic behavior of the ballast layer, it is difficult to predetermine which specific pairs of crushed stones among so many will come into contact with each other. It is also hard to predetermine where on the track or structures the wheelsets of a derailed vehicle would run and which part of the vehicle would come into contact with the track or structures. These issues highlight the importance of correctly identifying these 'contact pairs'.

As ballast can be regarded as granular material, a Dis- 
tinct Element Method (DEM), often used in the analysis of granular materials, is used for numerical simulation of its behavior. In the case of commonly used DEM, objects of the analysis are modeled as either polygonal elements or an aggregate of spherical elements that can move freely and the dynamic behavior of those elements is determined based on the analysis of their contact behavior. Many simulation programs use DEM algorism, each having its unique advantages and disadvantages over the others. Of these, RTRI uses two: one a discrete element model of a ballasted track, called DEMCS-track [7], developed jointly with Professor Matsushima of the University of Tsukuba. In this method, each crushed stone of ballast is represented by a combination of rigid spherical elements. The second method is a ballasted track simulator based on the Quadruple Discrete Element Method (QDEM) to analyze the elastic behavior of granular materials [8], developed jointly with the Japan Agency for Marine-Earth Science and Technology. This method is capable of analyzing ballast as granular material while factoring in the viscoelasticity of each crushed stone. The former method represents a relatively small computational cost and is therefore suitable for the initial assessment of various countermeasures against ballast settlement. The second method however is capable of analyzing stress distribution in each crushed stone of ballast (Fig. 3) and the load transfer associated with the elastoplasticity of each crushed stone of ballast. It is therefore suitable for the analysis of dynamic behavior of the ballasted tracks up to a high-frequency range. These methods are used selectively depending on the purpose of the analysis. In both methods, the distance between adjoining elements needs to be evaluated to identify appropriate contact pairs. The total number of pairs can be very high, requiring a considerable amount of time for computation. This shows the importance of an algorithm, or know-how, for efficiently identifying contact pairs.

On the numerical simulation of vehicle behavior after derailment in the case of a huge earthquake, RTRI developed a method for efficiently analyzing vehicle dynamic behavior after derailment [11] by improving the simulation program 'Dynamic Interaction Analysis for Shinkansen

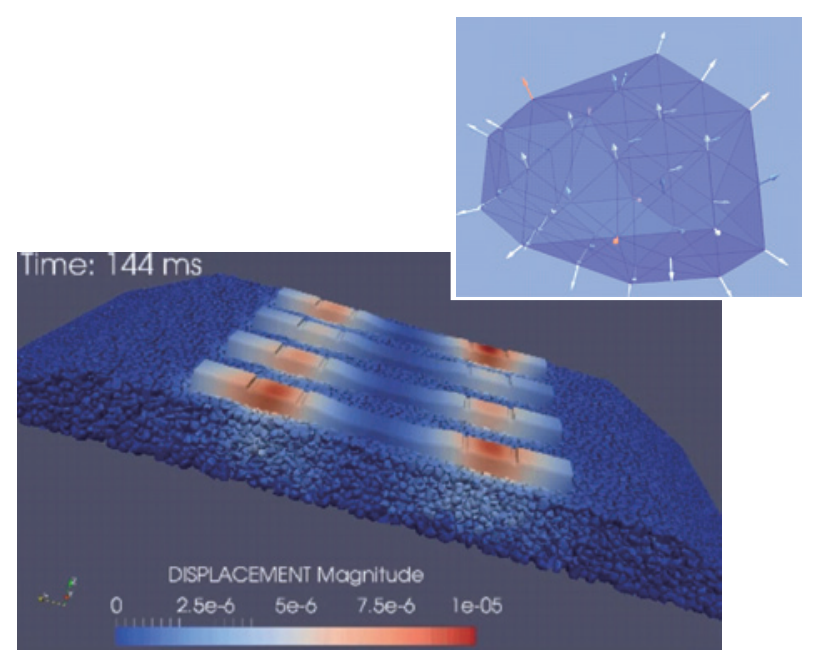

Fig. 3 Crushed stone model used in ballasted track simulator and calculated example of displacement distribution on a ballasted track

Train And Railway Structures (DIASTARS)' [12] developed by RTRI (Fig. 4). The improved method has the following features: the shapes of the vehicle-track or vehiclestructure contact areas after derailment are simplified; the numerous contact points are represented by a limited number of contact detection points; and characteristics of local deformation of each material are modeled as a contact spring. The method is capable of completing contact analyses efficiently and therefore can be used in various case studies in order to provide effective and concrete solutions against derailment.

\section{Contact wear analysis}

Rails and wheels are worn by rolling contact, while contact wires and contact strips are worn from sliding. When wear on a component exceeds a specific threshold, it has to be replaced. Accurate wear estimation is there-
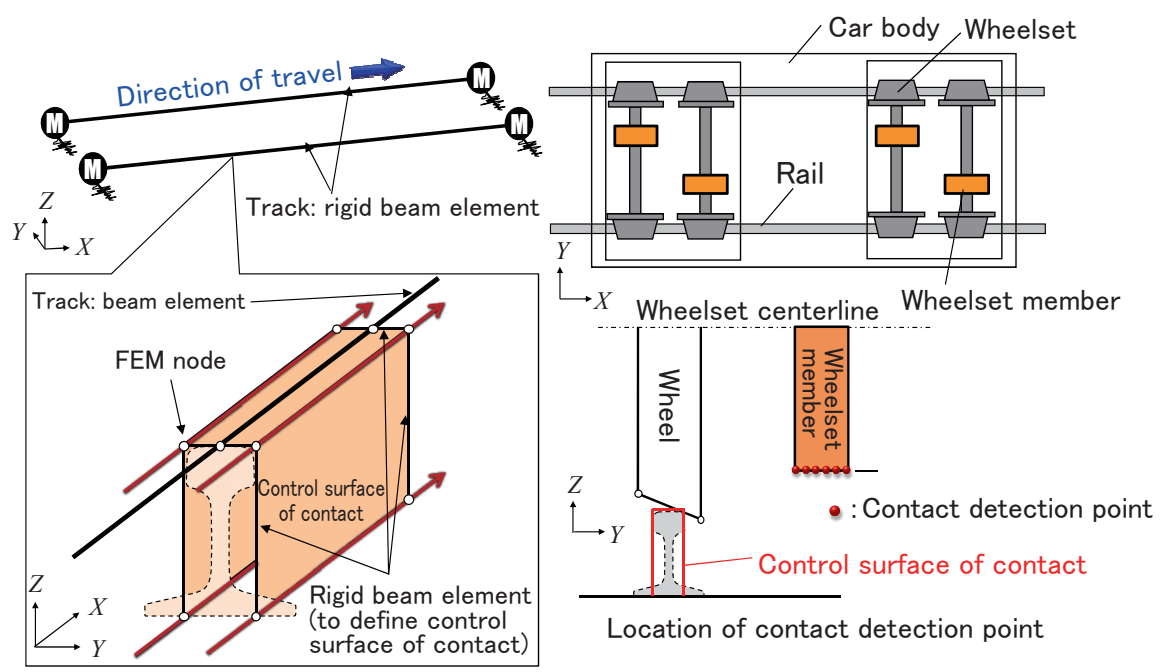

Fig. 4 Method for identifying contact points between wheelset members and track structures in the case of vehicle derailment 


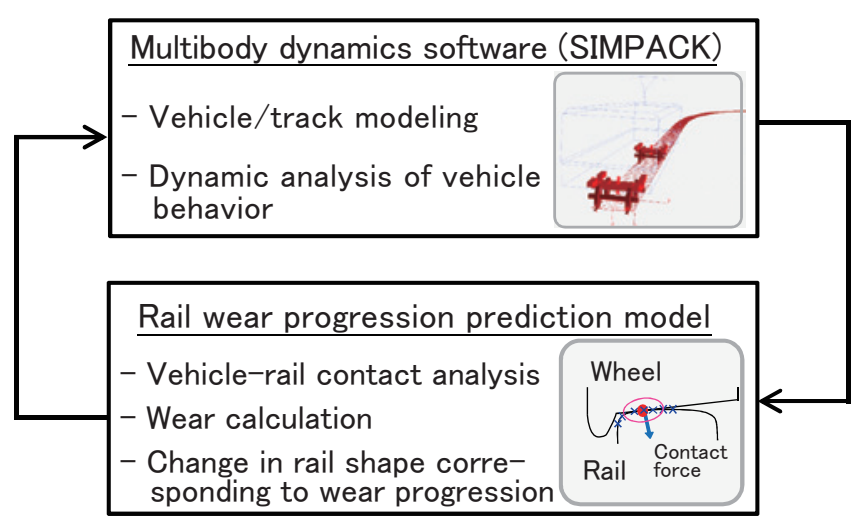

(a) Procedure of rail wear progression prediction
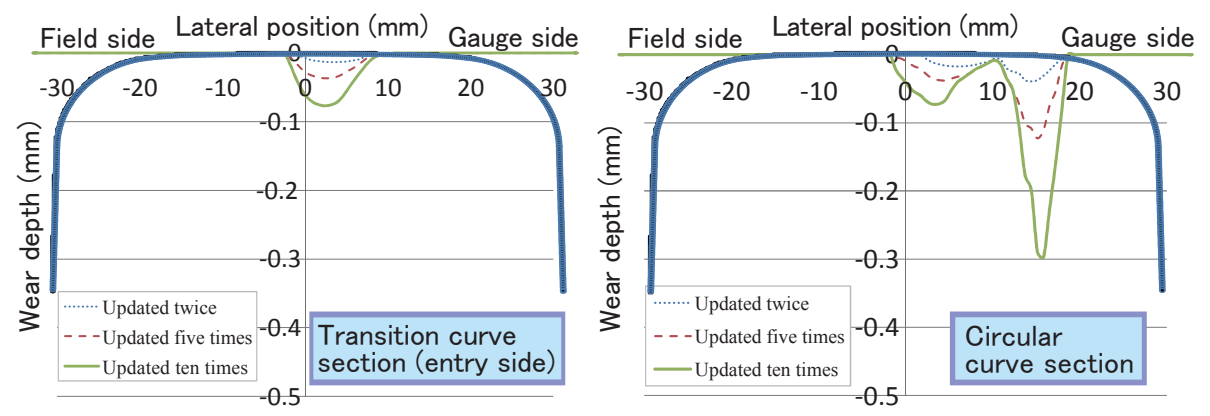

(b) Examples of numerical prediction of rail wear progression in curve sections

\section{Fig. 5 Method for prediction of rail wear progression by using multibody-dynamics analysis}

fore important for maintenance. Evaluating wear only through theoretical analysis however is difficult. Therefore, wear characteristics have traditionally been investigated through wear tests and wear is predicted by applying the appropriate wear model based on characteristics obtained through experimentation [13]. Nevertheless, quantitatively predicting wear progression on components solely through wear tests is complicated, for the following reasons: the rate of wear progression of rails/wheels and contact wire/ pantograph is heavily influenced by conditions of the environment in which the track and catenary are installed, the number of passing trains and the way the shape of the material changes with wear.

On this subject RTRI has been working in association with Professor Terumichi of Sophia University to develop a method for predicting wear progression in rails installed in various conditions while factoring in the dynamic behavior of passing trains and changes in shape of the rail surface as it wears [14-15]. In the method in question, the dynamic behavior of each wheel of a passing vehicle is analyzed using multibody dynamics. The contact force is then found through the analysis and the wear coefficient obtained in experiments is substituted into a wear progress equation in order to derive the degree of wear progression in the rail. As rail surfaces wear, rail-wheel contact conditions change. In order to take this into account, the rolling behavior of each wheel, following a certain degree of wear, is analyzed again with the same multibody dynamics approach. By repeating this process, rail wear progression can be predicted (Fig. 5). However, rail wear progression can be different depending on the characteristics of the passing vehicles (wheel shape, static wheel load, passing speed), variation of wear coefficients resulting from weather and other conditions, and other factors. A method is therefore needed to include these variables in an efficient and practical manner. A similar approach can also be applied to predict contact wire wear progression. There has been a study conducted overseas on this theme [16]. A major difference from rail wear prediction is that arcing damage due to contact loss needs to be considered.

\section{Conclusion}

This paper described RTRI's efforts in recent years to address issues related to contact phenomena on the railways. RTRI will continue to pursue this line of study on issues related to contact phenomena, which is a complex theme presenting many challenges, to improve railway safety and reliability.

\section{References}

[1] Ishida M., "Subjects on track technology focused on from tribological point of view," RTRI Report, Vol.22, No.8, pp.1-4, 2008, (in Japanese).

[2] Kalker J.J., Three-dimensional elastic bodies in rolling contact, Kluwer academic publishers, 1990.

[3] Sakai H., et.al., "Analysis of rolling contact behavior between wheel and rail through large-scale parallel computing," Quarterly Report of RTRI, Vol.55, No.3, pp.171-175, 2014.

[4] Hashimoto G., Okuda H., "Large-scale one-way cou- 
pled analysis with open-source parallel FEM software FrontISTR," NAFEMS European Conference on Multiphysics Simulation 2014, pp.31-33, 2014.

[5] Namura A., et.al., "Rail Surface Fatigue Assessment using numerical simulation of concurrence of short crack propagation and wear," Quarterly Report of RTRI, Vol.57, No.4, pp.317-317, 2016.

[6] Chen H., et.al., "Influence of Leaves on the Adhesion between Wheel and Rail," Quarterly Report of RTRI, Vol.59, No.1, pp.65-71, 2018.

[7] Kono A., Matsushima T., "3D-DEM simulation about dynamic behaviors of ballasted track under impact loading," Railway Engineering 2011, 2011.

[8] Nishiura D., et.al., "Novel discrete element modeling coupled with finite element method for investigating ballasted railway track dynamics," Computers and Geotechnics, pp.1-15, 2017.

[9] Miyamoto T., et.al, "Railway vehicle dynamic behavior against large-amplitude track vibration -A full-scale experiment and numerical simulation," Quarterly Report of RTRI, Vol.45, No.3, pp.111-115, 2004.

[10]Kuzuta M., et.al., "Experiments and simulation of guiding 1/10 scale model vehicle by guard force," $R T R I$
Report, Vol.28, No.12, pp.23-28, 2014, (in Japanese). [11] Goto K., et.al., "A Simple Method for Analyzing Contact between Wheelset Members and Track Structures Using MBD," Quarterly Report of RTRI, Vol.59, No.1, pp.57-64, 2018.

[12] Matsumoto N., et.al., "Running safety analysis of vehicles on structures subjected to earthquake motion," Quarterly Report of RTRI, Vol.45, No.3, pp116-122, 2004.

[13]Archard J.F., "Contact and rubbing of flat surface," $J$. Applied Science, Vol.24, pp.981-988, 1953.

[14] Yoshioka A., et.al., "Study on modeling and numerical analysis for the prediction of wheel wear development," Mechanical Engineering Journal, Vol.4, No.4, pp.17-00123-17-00126, 2017.

[15]Tsujie M., "A study on the development of a model for predicting worn profile of rail using multibody dynamics," First International Conference on Rail Transportation, 2017.

[16]Bucca G., Collina A., "Electromechanical interaction between carbon-based pantograph strip and copper contact wire: A heuristic wear model," Tribology International, Vol.92, pp.47-56, 2015.

\section{Author}

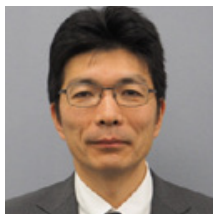

Mitsuru IKEDA

Director, Power Supply Technology Division (Formerly Railway Dynamics Division) Research Areas: Dynamic Interaction between Pantographs and Catenary Systems, OCS Maintenance, Pantograph Aerodynamic Noise Reduction 